\title{
MOTIVACIÓN LABORAL Y GESTIÓN DE RECURSOS HUMANOS EN LA TEORÍA DE FREDERICK HERZBERG
}

\author{
JULIO LÓPEZ MAS* \\ E-mail: johans_jlopez@yahoo.com
}

\begin{abstract}
«Todos debemos asumir la responsabilidad de conocernos a nosotros mismos para encontrar los empleos adecuados a medida que nos desarrollemos y la familia se convierte en un factor en sus valores y elecciones. (...).En la organización tradicional -la de los pasados cien años- el esqueleto, o la estructura interna, fue una combinación de jerarquía y poder. En la nueva organización debe ser comprensión y responsabilidad mutuas».
\end{abstract}

PETER DRUCKER (1993). El Ejecutivo Poscapitalista

\section{RESUMEN}

En la búsqueda de la competitividad empresarial en la Nueva Economía y Management los administradores deben diseñar estilos de liderazgo y practicas administrativas de alta eficiencia y desempeño de los recursos humanos. La complejidad de la motivación laboral requiere de enfoques que tomen en cuenta los factores personales y ambientales. La discusión de los aportes de un pensador pionero en el tema como Frederick Herzberg y la interpretación de un trabajo de tesis de maestría de nuestra Facultad de Ciencias administrativas titulado ; «Diseño y validación de un modelo para la identificación y medición de los factores motivacionales de los trabajadores según la teoría de F. Herzberg,» son las motivaciones de este trabajo reflexivo.

La practica y las investigaciones modernas de la administración señalan a la motivación como factor clave que los gerentes incorporan en las relaciones de trabajo que crean y supervisan. La función administrativa de la Dirección se entiende, como el proceso de influir en la gente, para que contribuya a los objetivos de los individuos y de la organización en conjunto; para esto se requiere la creación y el mantenimiento de un contexto en el que los individuos trabajen juntos en grupos hacia el logro de objetivos comunes ${ }^{1}$.

Palabras clave: Motivación, satisfacción, administración de recursos humanos.

* Licenciado en Economía Política. Doctor en Economía e Historia Latinoamericana de la Universidad de Paris-EHESS; Profesor Principal de la Facultad de Ciencias Administrativas de la Universidad Nacional Mayor de San Marcos. Actualmente es Jefe de la Oficina de Calidad Académica y Acreditación de la FCA. Es Asesor de Foncodes en temas de Gerencia Social y Descentralización. 


\begin{abstract}
In the search for the industry competitivity in the New Economy and Management the administrators must design leadership styles and administartive practices of high eficiency and perfomance of human resources. The complexity of the laboral motivation requires approaches that consider personal and enviromental factors. The argumentation on the contributions of a pioneer thinker on this subject like Frederick Herzberg and the interpretation of a tesis work for Magister Degree on our «Facultad de Ciencias Administrativas» intitled : «Diseño y validación de un modelo para la identificación y medición de los factores motivacionales de los trabajadores según la teoría de F. Herzberg», are the motivations on this reflexive article.

The practice and modern investigations on management show the motivation as a decisive factor that modern managers include in work relationships that they create and monitorize.

The administrative function of the Management is understood as the procedure that influences on people, in order to contribute to achieve the objetives of the individuals and the organization jointly; for this is required to create and the sustenance of a context in which the individuals work together in groups to achieve common objetives ${ }^{1}$.
\end{abstract}

Keywords: Motivation, satisfaction, human resources administration. ¿QUÉ ES LA MOTIVACIÓN?

Las Ciencias del Comportamiento nos ayudan a conocer y aprovechar los factores humanos y los relativos a la motivación. ¿Qué entendemos por motivación? La motivación es una característica de la psicología humana que contribuye al grado de compromiso de la persona; es un proceso que ocasiona, activa, orienta, dinamiza y mantiene el comportamiento de los individuos hacia la realización de objetivos esperados $^{2}$. A pesar que los patrones de comportamiento varían (necesidades de individuo, valores sociales y capacidad individual) el proceso es el mismo para todas las personas: el comportamiento es causado (causa interna o externa, producto de la herencia y/o del medio ambiente); el comportamiento es motivado, ya sea por impulsos, deseos, necesidades o tendencias, y el comportamiento está orientado, siempre está dirigido hacia algún objetivo.

Debemos añadir que «no existe la persona promedio». Los individuos son únicos: tienen distintas necesidades, distintas ambiciones, distintas actitudes, distintos deseos en cuanto a la responsabilidad, distintos niveles de conocimiento y habilidades así como distintos potenciales. Hay que entender la complejidad y la singularidad de las personas ${ }^{3}$.

La motivación, dentro del ámbito laboral, es definida actualmente como un proceso que activa, orienta, dinamiza y mantiene el comportamiento de los individuos hacia la realización de objetivos esperados.
Por esto, en el ámbito laboral es importante conocer las causas que estimulan la acción humana, ya que mediante el manejo de la motivación, entre otros aspectos, los administradores pueden operar estos elementos a fin de que su organización funcione adecuadamente y los miembros se sientan más satisfechos.

Una carencia importante en el contexto laboral peruano es que no se cuenta con modelos e instrumentos válidos para la medición de la motivación, por lo que es meritoria la investigación realizada por el Mag. Walter Arana, graduado de nuestro Programa de Maestría en Administración, quien ha sustentado y obtenido el grado de Magíster con la tesis titulada «Diseño y validación de un modelo para la identificación y medición de los factores motivacionales de los trabajadores según la teoría de Frederick Herzberg» ${ }^{4}$. Como es por todos conocidos F. Herzberg es un autor importante en el desarrollo del pensamiento administrativo moderno; sus investigaciones y las de sus seguidores han formulado la denominada Teoría Bifactorial o de los Dos factores sobre la motivación y con este aporte el enfoque de la Jerarquía de las Necesidades de Maslow ha sido considerablemente modificado ${ }^{5}$.

La investigación de tipo aplicativa del Mag. W. Arana ha tenido como producto la elaboración de un modelo destinado a servir de herramienta de gestión de los recursos humanos en el ámbito empresarial que permite identificar y medir la sustracción, lanzando un test basado 
en el modelo de F. Herzberg. El sustento empírico se basa en un trabajo de campo realizado con una muestra de empleados de empresas localizadas en Lima y Huancayo ${ }^{6}$.

El presente artículo tiene como propósito profundizar el entendimiento de la teoría Bifactorial de Frederick Herzberg y presentar al respecto la visión actual de la motivación y satisfacción laboral a la luz del pensamiento administrativo reciente y del nuevo management. Asimismo, proponemos una interpretación de los resultados de la investigación pionera del Mag. W. Arana.

Por lo tanto, ponemos a discusión la hipótesis de F. Herzberg de que únicamente el logro de los resultados intrínsecos motivadores del trabajo puede iniciar una motivación sostenida hacia los objetivos de la organización.

\section{TEORÍAS DE LA MOTIVACIÓN}

Existen muchas teorías de la motivación; cada una de ellas explica, en cierta medida, lo que las personas piensan les resulta importante y que está ocurriendo en su derredor. Las teorías de la motivación difieren en cuanto al factor que consideran tiene mayor importancia para lograr la motivación, y con esta base realizan los pronósticos adecuados. La Teoría de las Necesidades y la Teoría de la Equidad se refieren a las satisfacciones e insatisfacciones de las personas. La teoría de los Refuerzos se refiere a que las consecuencias de una conducta específica pueden afectar su repetición. La Teoría de las Expectativas detalla el proceso mediante el cual las personas pueden optar por distintas acciones alternativas, en base a sus expectativas de lo que obtendrán de cada conducta. La Teoría de las Metas se concentra en el proceso de establecerlas y la forma en que las metas mismas afectan la motivación. No obstante, estas cinco posiciones tienen en común el papel crucial de la conciencia de la persona en cuanto a lo que le resulta importante y las circunstancias en las que trabaja ${ }^{7}$.

\section{TEORÍA DE LAS NECESIDADES}

De acuerdo con la teoría de las necesidades, una persona está motivada cuando todavía no ha alcanzado ciertos grados de satisfacción en su vida. Una necesidad satisfecha no es motivadora. Esta teoría se refiere a aquello que necesitan o requieren las personas para llevar vidas gratificantes, en particular con relación a su trabajo.

Existen diversas teorías de las necesidades, las mismas que difieren en cuanto a los grados y el punto en que, de hecho, se alcanza la satisfacción. Entre las más importantes tenemos la Jerarquía de las Necesidades de Maslow; las Tres Necesidades de David C. McClelland, y la Teoría de los Dos Factores de la Motivación de F. Herzberg.

Según la opinión de Stanton y otros ${ }^{8}$

«cada una de las teorías de las necesidades... subraya la satisfacción de algunas necesidades particulares importantes que las personas han conseguido con el tiempo. Cada una de las teorías también destaca que las personas deciden cuál es el grado de satisfacción, comparando, conscientemente sus circunstancias y sus necesidades. Por último, cada teoría da cabida a una variación considerable de una persona a otra, y 'en' una misma persona, con el tiempo».

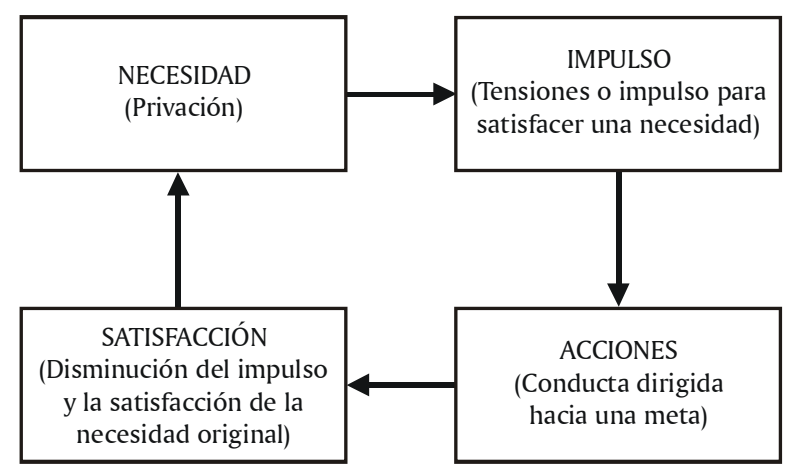

\section{LA TEORÍA DE LA JERARQUÍA DE LAS NECESI- DADES}

Una de las teorías sobre motivación más ampliamente mencionada es la Teoría de la Jerarquía de las Necesidades, establecida por el psicólogo Abraham Maslow, partir de observaciones clínicas (Maslow, 1943). Este autor propone que la motivación humana se basa en la voluntad de satisfacer sus necesidades (fuerza interna), identificando una jerarquía de cinco necesidades, desde las necesidades fisiológicas básicas hasta las necesidades más altas de realización personal. Las cinco necesidades son necesidades fisiológicas, necesidades de seguridad, necesidades de pertinencia, necesidades de estima y necesidades de autorrealización. 


\section{JERARQUÍA DE LAS NECESIDADES DE MASLOW}

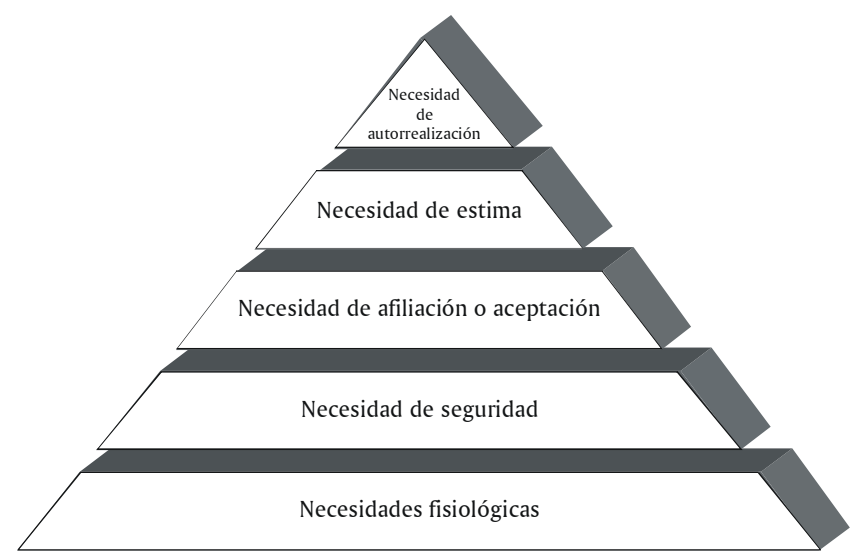

Según Maslow, las personas tendrán motivos para satisfacer cualesquiera de las necesidades que les resulten más predominantes o poderosas en un momento dado. El predominio de una necesidad dependerá de la situación presente de la persona y de sus experiencias recientes. A partir de las necesidades fisiológicas, que son las básicas, se debe ir satisfaciendo cada necesidad, antes de que la persona desee satisfacer la necesidad del siguiente nivel superior.

Un resumen de sus principales planteamientos lo encontramos en su artículo «Una Teoría de la Motivación Humana» ${ }^{9}$, que presentamos a continuación:

a) Hay por lo menos cinco conjuntos de objetivos que pueden denominarse necesidades básicas, son las necesidades fisiológicas, de seguridad, de amor, de estima y de realización personal. Además, estamos motivados por el deseo de conseguir o mantener las condiciones que aseguran estas satisfacciones básicas y por algunos deseos mas intelectuales ${ }^{10}$.

b) Estos objetivos básicos están interrelacionados y ordenados en una jerarquía de prioridades. Estos significa que el objetivo prioritario monopolizará el conocimiento consciente y tenderá, en sí mismo, a organizar la activación de las diversas capacidades del organismo. Las necesidades menos predominantes se minimizan, incluso pueden olvidarse o negarse. Pero cuando una necesidad está suficientemente satisfecha, surge la siguiente necesidad (superior) prioritaria que, a su vez, dominará la vida consciente y actuará como centro organizador de la conducta, ya que las necesidades satisfechas dejan de ser motivadores activos ${ }^{11}$. c) Grado de satisfacción relativa. Se puede dar la impresión de que los cinco grupos de necesidades tienen entre sí una relación escalonada, de todo o nada. Hemos hablado en términos parecidos a esto: "si se satisface una necesidad, entonces surge otra». Esta afirmación puede producir la falsa impresión de que una necesidad tiene que satisfacerse al ciento por ciento antes de que surja la próxima. En la vida real, la mayoría de miembros normales de nuestra sociedad están parcialmente satisfechos y parcialmente insatisfechos en todas sus necesidades básicas al mismo tiempo ${ }^{12}$.

d) Función de las necesidades satisfechas. Si nos interesa lo que de verdad nos motiva y no lo que nos ha motivado o nos motivará, entonces una necesidad satisfecha no es un elemento motivador y para todos los fines prácticos debe considerarse que no existe, que ha desaparecido ${ }^{13}$.

e) Necesidad de Realización Personal.

"...diría sencillamente que un hombre sano está motivado principalmente por sus necesidades de desarrollar y realizar todo su potencial y toda su capacidad»... "se refiere al deseo de cumplir nuestros deseos más profundos, es decir, hacer realidad lo que somos en potencia... es el deseo de ser cada vez más lo que uno es, de llegar a ser lo que uno es capaz de llegar a ser» ${ }^{14}$.

Siendo importante y sugerentes estos planteamientos, sin embargo existe la apreciación actual de que la Jerarquía de Necesidades de Maslow no describe un proceso de motivación universal humano sino que, más bien, sería la descripción de un sistema de valores específico de la clase media de Estados Unidos. Otros estudios de carácter empírico, como el de Lawler y Suttle ${ }^{15}$, aplicados a 187 administradores en dos organizaciones distintas durante un periodo de 6 a 12 meses, encontraron evidencia empírica de la existencia de dos niveles de necesidades biológicas y otras, y que las necesidades biológicas emergen solo cuando éstas no se ven razonablemente satisfechas, lo cual puede ser congruente con la idea del grado de satisfacción relativa que hemos dicho anteriormente. Además, encontraron que, en un nivel superior, la fuerza de las necesidades variaba de acuerdo con el individuo; en ciertos individuos, las necesidades sociales predominaron, mientras que, en otros, las necesidades de autorrealización fueron más fuertes. 
Estas conclusiones refuerzan la idea de la complejidad y singularidad de las personas.

Otra perspectiva de análisis se basa en las diferencias culturales y sociales. Según los estudios de Geert Hofstede ${ }^{15}$, la cultura se relaciona con los valores laborales. Como parte de su trabajo como psicólogo de la IBM, Hofstede recopiló datos relativos a los valores y las actitudes del empleado en aproximadamente 100,000 individuos de 1967 a 1973. Estos datos le permitieron comparar factores culturales entre 40 países y llegó a ciertas conclusiones sobre la relación entre el carácter nacional y la motivación de los empleados. Hofstede se refiere a cuatro dimensiones que, en su opinión, describen aspectos importantes de una cultura nacional. El individualismo contrapuesto al colectivismo mide la relación de una persona con otras, así como el deseo de libertad personal en comparación con la necesidad de tener nexos sociales. La distancia del poder evalúa la forma en que una sociedad concreta maneja la desigualdad de las personas. La evasión de la incertidumbre mide la forma en que una sociedad enfrenta la incertidumbre del futuro. La masculinidad contrapuesta a la feminidad se refiere a la rigidez de los roles de los sexos. Por lo tanto, se podría concluir también que el orden de importancia de las necesidades difiere de un país a otro. Algunas personas trabajan mucho más de lo que sería adecuado para satisfacer sus necesidades básicas. La importancia relativa del trabajo está determinada en gran medida por la relación de los ámbitos cultural y económico. En igual sentido, ocupaciones diferentes proporcionan retribuciones económicas, sociales y de prestigio diferentes en países diferentes ${ }^{16}$.

Otro investigador, Clayton Alderfer, desarrolla la llamada Teoría ERG, con similitudes a la de la Jerarquía de Necesidades, pero consideraba que las necesidades (fuerzas internas) se podían agrupar en tres categorías: las necesidades existenciales (las necesidades fundamentales de Maslow), las necesidades de relación (necesidades de relaciones interpersonales) y las necesidades de crecimiento (necesidades de creatividad personal). Además, da un énfasis particular a un aspecto que lo diferencia de la teoría de Maslow en el sentido de que una persona puede estar motivada por necesidades de distintos niveles a un mismo tiempo y el grado de motivación dependerá de la intensidad de la necesidad ${ }^{17}$.

\section{TEORÍA BIFACTORIAL DE HERZBERG}

En su artículo «Una vez más: ¿Cómo se motiva a los empleados?» Frederick Herzberg ${ }^{18}$ plantea que la psicología de la motivación es tremendamente compleja y reconoce que lo descifrado con un cierto grado de seguridad es realmente poco, que existe una enorme desproporción entre conocimiento y especulación, requiriéndose «ideas que han sido puestas a prueba en numerosas empresas y otras organizaciones», y este enfoque es su aporte.

\section{Satisfacción e insatisfacción laboral}

«Es necesario un breve repaso a mi teoría de motivación-higiene en las actitudes en el trabajo antes de poder ofrecer sugerencias teóricas y practicas. La teoría surgió de un examen de ciertos sucesos en la vida de ingenieros y contables. Desde entonces se han completado por lo menos otras 16 investigaciones, utilizando una extensa variedad de sujetos (incluyendo algunos en los países comunistas), haciendo de aquel trabajo original uno de los estudios más repetidos en el campo de las actitudes laborales».

Lo descubierto por estos estudios, corroborado por muchas otras investigaciones que utilizaron procedimientos diferentes, sugiere que los factores implicados en producir satisfacción (y motivación) en el trabajo son algo separado y distinto de aquellos que llevan a la insatisfacción en el trabajo.

Puesto que tenemos que considerar factores separados, dependiendo de si analizamos la satisfacción o la insatisfacción en el trabajo, se deduce que estos dos sentimientos no son opuestos entre sí. Lo opuesto a satisfacción en el trabajo no es insatisfacción sino, en cambio, falta de satisfacción y, de forma pareja, lo opuesto de insatisfacción no es satisfacción, sino falta de insatisfacción ${ }^{19}$.

La teoria bifactorial de Herzberg afirma que la motivación se genera por la búsqueda de una satisfacción optima de ciertas necesidades, las que producen satisfacción laboral ${ }^{20}$.

\section{Dos necesidades diferentes de la persona}

«Podemos decir que un conjunto de necesidades se derivan de nuestra naturaleza animal: el instinto que nos lleva a evitar cualquier dolor causado por el medio ambiente, suma- 
do a todos los móviles que adquirimos y que llegan a estar condicionados a las necesidades biológicas básicas.(..). El otro conjunto de necesidades se refiere a esa característica singular del ser humano, la capacidad de logro, y por medio de ese logro, de conseguir el desarrollo psicológico.

Los estímulos para ese desarrollo son las tareas que lo inducen: en un contexto industrial, son el contenido del trabajo. A la inversa, los estímulos que fomentan una conducta encaminada a evitar el dolor se encuentran en el entorno laboral» ${ }^{21}$.

Sin embargo, en la realidad industrial sigue siendo una preocupación gerencial el desarrollo de la motivación intrínseca, pero se requiere revisar las creencias acerca de las personas en la sociedad industrial contemporánea (la gente solo trabaja para lograr beneficios materiales; la gente solo trabaja por la remuneración); y en ese sentido es valida la direccionalidad señalada por Herzberg. Para este propósito, las organizaciones (y empresas) actuales adoptan la disciplina de la innovación. Con los nuevos paradigmas de la economía del conocimiento las organizaciones deben aprender lo necesario para ser eficaces e innovar, para hacer cosas nuevas es necesario contar con personas altamente motivadas y constituidas como comunidades (conjunto de individuos que comparten un propósito común). Sabemos que la búsqueda de la realización personal y la fidelidad con los propósitos procuran placer en el trabajo: estimulan la pasión, la paciencia y la perseverancia, imprescindibles para crecer como persona y en el ámbito de la organización. Sin embargo, en el ámbito industrial y laboral la gente es incapaz de definir resultados relacionados con sus pasiones más hondas, a menos que los líderes cultiven un ambiente donde esas pasiones se puedan articular sin peligro. Muchos tienen talento, pero el aprendizaje real requiere disciplina, proceso mediante el cual logramos nuestro pleno potencial gracias al compromiso, la pasión, la paciencia y la perseverancia ${ }^{22}$.

\section{Factores motivadores y de higiene}

"Los factores de desarrollo o motivadores intrínsecos al trabajo son: realización, el trabajo mismo, responsabilidad y progreso o desarrollo. Los factores que llevan a evitar la insatisfacción o factores de higiene extrínsecos al trabajo incluyen: la política y la administración, supervisión, relaciones interpersonales, condiciones de trabajo, salario, nivel laboral y seguridad vigentes en la empresa» ${ }^{23}$.

En las investigaciones recientes se ha tipificado el entorno de la organización, el que posee cuatro características que pueden servir de fuentes de resultados motivacionales a diferencia de enfoques como la teoría de Herzberg ${ }^{24}$ y asimismo, el medio ambiente en general distingue el medio ambiente externo del medio ambiente interno; en el primero, la eficacia se considera como el principal elemento para evaluar la relación entre la organización y el medio ambiente, y en el segundo, para incrementar la eficiencia se requiere utilizar la responsabilidad colectiva y el espíritu de cooperación ${ }^{25}$.

\section{$Y$ en general:}

- El trabajo mismo puede proporcionar resultados intrínsecos o a través de las tareas.

- Las políticas y prácticas de personal referidas a las recompensas, constituyen una fuente de resultados.

- Las relaciones interpersonales con el supervisor y la medida en que éste reconozca los logros del individuo y le permita participar, son también fuentes de resultados.

- Los compañeros de trabajo son habitualmente una fuente de resultados.

Para emprender un nuevo diseño organizacional se deben considerar cuatro dimensiones de cambio: personal, interpersonal, de dirección y de organización. Se trata de «un amplio ecosistema interrelacionado e interdependiente» ${ }^{26}$.

\section{Base empírica de la teoría}

En sus trabajos iniciales (fines de años 50), Herzberg y sus colaboradores realizaron un estudio de la actitud laboral de 200 ingenieros y contadores; para 1969 se habían realizados doce estudios.

«...En el cuadro: Factores que afectan a las actitudes en el trabajo, según datos recogidos en 12 estudios, muestro el conjunto de los factores que causan satisfacción e insatisfacción en el trabajo, obtenidos de una muestra de 1685 empleados. A los empleados se les preguntó que acontecimientos en sus puestos 
de trabajo les habían producido una satisfacción o insatisfacción extrema.

Los resultados indican que los motivadores $(81 \%$ de los factores de satisfacción) fueron la causa principal de satisfacción y los factores de higiene (69\% de los factores de insatisfacción) son la causa principal de infelicidad en el trabajo» ${ }^{27}$.

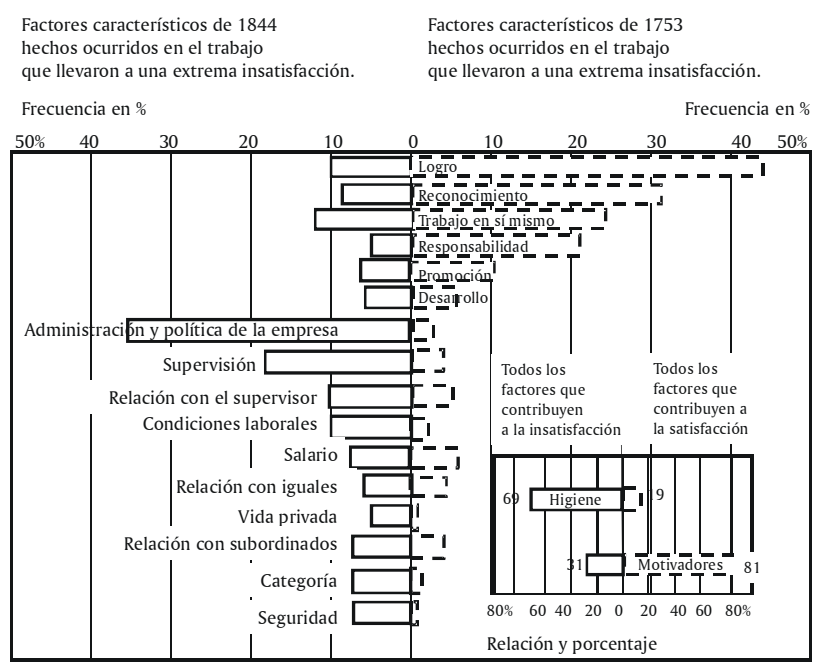

Factores que afectan a las actitudes en el trabajo, según datos recogidos en 12 estudios. Fuente: F. Herzberg (1969)

Sin embargo, existe una variedad de estudios empíricos, algunos críticos y otros confirmatorios.

\section{Eficacia de la Teoría de la Higiene-Motivación}

«La teoría de la Higiene- Motivación (..) sugiere que el trabajo debe enriquecerse para conseguir una eficaz utilización del personal. (...) El enriquecimiento de la tarea ofrece al empleado la oportunidad de desarrollarse psicológicamente... este artículo se limita a sugerir aquellos principios y medidas prácticas que se deducen de diversos experimentos realizados con éxito en la industria» ${ }^{28}$.

Es lo que denomina F. Herzberg como el desarrollo vertical del puesto de trabajo mediante la creación de factores motivadores.

En la época actual -donde las empresas luchan por transitar de una economía basada en la producción a otra fundada en el conocimiento, en la que la creación del valor depende cada vez más de las ideas y la innovación-, ya no es suficiente el enriquecimiento del puesto. Actualmente se diseñan diversas herramientas administrativas para propiciar actitudes y comportamientos indispensables para el alto desempeño: generar confianza, desencadenar nuevas ideas, y asimismo obtener la cooperación voluntaria de los empleados implicados con procesos y resultados que también les favorezcan en su desarrollo personal ${ }^{29}$. Y asimismo, también debería remarcarse que los directivos del management deben desarrollar su motivación intrínseca, con énfasis en la responsabilidad, desarrollo, trabajo mismo, etc., y no ser solo considerado como un factor de acompañamiento, de soporte al trabajo operativo de los empleados.

El enfoque del enriquecimiento del puesto fue retomado por el sistema socio-técnico del trabajo $^{30}$, el cual integra una visión colectiva del trabajo y una noción de interrelación (al interior de un grupo de trabajo y de éste con el resto de la organización). Con este enfoque sistémico de las organizaciones y del nuevo management, se plantea la insuficiencia del análisis psicológico, que considera al individuo en el trabajo sin tener en cuenta las relaciones estructurales (relaciones laborales) y de poder (entorno organizacional, tipo de supervisión, organización del trabajo, cultura organizacional, etc.). Para otros autores, el servicio comunitario es una forma de enriquecer el trabajo, en la medida que

«los estudios confirman que los programas de voluntariado aumentan la moral de los empleados, su lealtad y su productividad, todo cual contribuye a aumentar el rendimiento profesional» ${ }^{31}$.

Con este planteamiento la gestión de los recursos humanos se ve beneficiada con estas formas de servicio comunitario que contribuirían al desarrollo profesional y a lograr un efectivo compromiso con la comunidad.

\section{Función de la Dirección de Personal}

Sabemos que el reto de la Dirección es lograr los resultados deseados, con eficiencia, calidad e innovación, y la motivación laboral es un factor básico para el rendimiento eficiente que se espera. Con este propósito presentamos la teoría de Herzberg sobre el enriquecimiento del trabajo. ¿En que consiste?, ¿crea las condiciones adecuada para que aflore el comportamiento deseado?

«El enriquecimiento de un puesto de trabajo no puede ser una propuesta de un momento dado, sino una función continuada de dirección. No 
obstante, los cambios iniciales deberían durar un largo periodo de tiempo.

Diversas razones para ello:

- Los cambios deberían elevar el trabajo hasta un nivel de dificultad equiparable a la capacidad exigida al efectuar la contratación de un empleado;

- Aquellos que tengan aún más conocimientos, podrán demostrarlos mejor y conseguir la promoción a un puesto de más alto nivel;

- La naturaleza misma de los motivadores, a diferencia de los factores de higiene, es que tienen un efecto a mucho más largo plazo en las actitudes de los empleados. Puede que haya que enriquecer el trabajo de nuevo, pero esto no sucederá con tanta frecuencia como en el caso de los factores de higiene» ${ }^{32}$.

\begin{tabular}{|c|c|}
\hline Principio & Motivador implicado \\
\hline $\begin{array}{l}\text { A Suprimir controles pero manteniendo } \\
\text { responsabilidad. }\end{array}$ & $\begin{array}{l}\text { Responsabilidad y sentido del } \\
\text { logro. }\end{array}$ \\
\hline $\begin{array}{l}\text { B Aumentar la responsabilidad del individuo } \\
\text { por su propio trabajo. }\end{array}$ & $\begin{array}{l}\text { Responsabilidad y } \\
\text { reconocimiento. }\end{array}$ \\
\hline $\begin{array}{l}\text { C Dar a la persona una unidad natural de trabajo } \\
\text { completa (módulo, división, área, etc.). }\end{array}$ & $\begin{array}{l}\text { Responsabilidad, sentido del } \\
\text { logro, reconocimiento. }\end{array}$ \\
\hline $\begin{array}{l}\text { D Conceder autoridad adicional a un empleado } \\
\text { en su actividad, libertad en el trabajo. }\end{array}$ & $\begin{array}{l}\text { Responsabilidad, sentido del } \\
\text { logro, reconocimiento. }\end{array}$ \\
\hline $\begin{array}{l}\text { E Poner a disposición del empleado } \\
\text { directamente, en lugar de a través del } \\
\text { supervisor, informes periódicos sobre su trabajo. }\end{array}$ & Reconocimiento interno. \\
\hline $\begin{array}{l}\text { F Introducir tareas nuevas y más difíciles nunca } \\
\text { desempeñadas antes. }\end{array}$ & Desarrollo y aprendizaje. \\
\hline $\begin{array}{l}\text { G Asignar a un individuo tareas específicas } \\
\text { o especializadas, que les permitan llegar } \\
\text { a ser especialistas. }\end{array}$ & $\begin{array}{l}\text { Responsabilidad, desarrollo } \\
\text { y promoción. }\end{array}$ \\
\hline
\end{tabular}

Principios del desarrollo vertical del puesto de trabajo Fuente: F. Herzberg (1969)

El enriquecimiento de puestos se orienta a crear trabajos que sean desafiantes y significativos, mediante el estímulo de factores intrínsecos de motivación como el reto, el reconocimiento de logro y la responsabilidad.

En la evaluación de los programas empresariales se evidenciaron varias limitaciones en su aplicación ${ }^{33}$. Así tenemos que el enriquecimiento del puesto de trabajo son técnicas para ampliar el contenido de los puestos de trabajo, introduciendo nuevas funciones y/o responsabilidades. El desarrollo vertical del puesto propuesto por Herzberg fue aplicado durante las décadas de 1970 y 1980 en muchas empresas, de gran prestigio como ITT, Sherwin-Williams, IBM, General Electric en Estados Unidos y Olivetti, Volvo, Phillips en otros países. Los resultados fueron variados: algunos han tenido éxitos notables, otros programas han sido menos satisfactorios, y estos resultados se derivan de las dificultades de su aplicación, al requerirse una gran cantidad de tiempo, paciencia, apoyo de seguimiento, y en general, una política de apoyo a estos programas de largo plazo, y una gran coordinación con otras funciones de la organización como los sistemas de evaluación de puestos de trabajo y remuneración, sistemas de planificación de la trayectoria profesional, programas de evaluación de supervisores y directivos y mejoras en los sistemas de datos y tecnología de la maquinaria.

Una concepción más actual relacionada con el enriquecimiento de puestos de Herzberg es el enfoque de Calidad de la Vida Laboral ${ }^{34}$, éste contiene una propuesta sistémica aplicada al diseño de puestos, combinada con una cimentación en el enfoque socio técnico, que como hemos indicado anteriormente combina sicología y sociología industriales y de la organización, diseño industrial, teoría y desarrollo de organización, teoría de la motivación y liderazgo y relaciones industriales. Este es un enfoque interesante, actualmente hay cientos de estudios modelos y programas prácticos, principalmente en Gran Bretaña y Escandinavia.

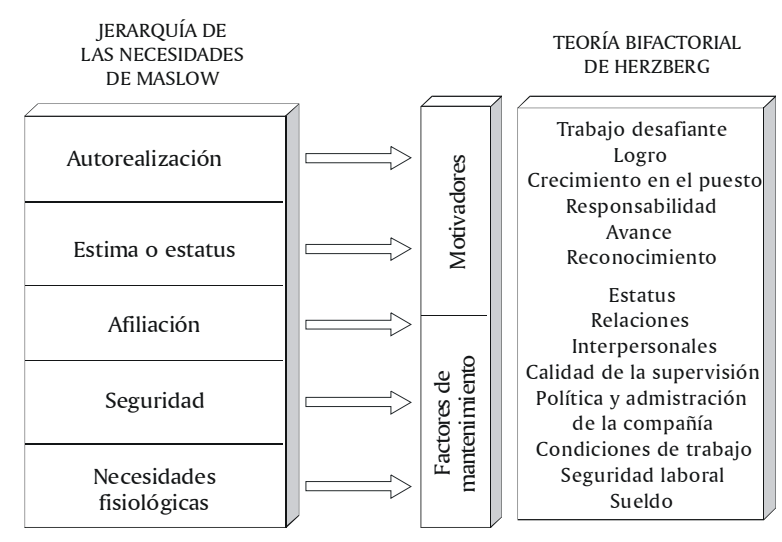

\section{DIAGNÓSTICO DE FACTORES MOTIVACIONALES EN EMPLEADOS PERUANOS}

El punto de partida es la opinión que las diferentes teorías sobre motivación no siempre son enteramente aplicables a todas las realidades y en todos los países. Dependerá de la cultura, costumbres, valores, situaciones sociales y económicas que condicionarán el modo de pensar y actuar de los trabajadores, situación que incide en los estudios que se realiza con relación a la motivación ${ }^{35}$. Como hemos argumentado anteriormente, es necesario tomar en cuen- 
ta tanto la complejidad y singularidad de cada persona así como las condiciones sociales y culturales diferentes y cambiantes a nivel nacional e internacional.

Por lo tanto, en toda investigación relacionada con la motivación laboral no se deberían aplicar genéricamente los conceptos teóricos existentes, sino que éstos deben ser analizados en función de cada sociedad o cultura, para diagnosticar cuidadosamente las necesidades de los trabajadores antes de preparar e implementar un plan de motivación ${ }^{36}$.

La tesis de Maestría del Mag. Walter Arana que estamos analizando -como hemos indicado- se propuso identificar y medir los factores motivacionales de los trabajadores peruanos según la teoría de F. Herzberg, para lo que se estimó conveniente hacer el estudio de trabajadores (empleados) pertenecientes a empresas ubicadas en la provincia de Lima, donde se encuentran el $62.33 \%$ del total nacional de las empresas de producción, comerciales y de servicios y en la provincia de Huancayo, por su condición de ser una provincia cosmopolita, un prototipo de la industria y comercio provinciana y donde se localiza el $2.24 \%$ del total de empresas del país. Asimismo, se decidió comprender a los trabajadores de la categoría ocupacional de empleados. Por la naturaleza de la investigación, se determinó usar una muestra intencionada no probabilística en función de las autorizaciones obtenidas en las empresas para la evaluación de personal así como una muestra de sujetos voluntarios... En suma, la población considerada en la investigación comprendió a 1,232 trabajadores (82.6\% de la provincia de Lima y $17.40 \%$ de la provincia de Huancayo $)^{37}$.

Los resultados de la investigación de campo señalan que: 1) Los factores motivadores: responsabilidad, trabajo en sí mismo, crecimiento, reconocimiento y promoción presentan correlación positiva muy fuerte. La correlación del factor logro es positiva en forma considerable. 2) Los factores higiénicos: seguridad, relación con el jefe, supervisión, condiciones de trabajo, sueldo, relación con los compañeros, vida personal, y política y administración, tienen correlación muy fuerte y la correlación del factor estatus es positivamente considerable. 3) Los factores relación con los subordinados y poder obtuvieron una correlación baja o débil, lo que significa que para los evaluados estos factores no son rele- vantes como elementos motivadores, resultado que contrasta con la teoría de Frederick Herzberg y David MacClelland ${ }^{38}$.

\begin{tabular}{|ll|}
\hline \multicolumn{2}{|c|}{$\begin{array}{c}\text { CORRELACIÓN DE PEARSON DE LOS } \\
\text { FACTORES MOTIVADORES }\end{array}$} \\
\hline Responsabilidad & $\mathrm{r}=0.99$ Positiva muy fuerte \\
\hline Trabajo en si mismo & $\mathrm{r}=0.98$ Positiva muy fuerte \\
\hline Crecimiento & $\mathrm{r}=0.98$ Positiva muy fuerte \\
\hline Reconocimiento & $\mathrm{r}=0.97$ Positiva muy fuerte \\
\hline Promoción & $\mathrm{r}=0.97$ Positiva muy fuerte \\
\hline Logro & $\mathrm{r}=0.87$ Positiva considerable \\
\hline & $\mathrm{r}=0.97$ Positiva muy fuerte \\
\hline Seguridad & $\mathrm{r}=0.97$ Positiva muy fuerte \\
\hline Relación con el jefe & $\mathrm{r}=0.96$ Positiva muy fuerte \\
\hline Supervisión & $\mathrm{r}=0.96$ Positiva muy fuerte \\
\hline Condiciones de trabajo & $\mathrm{r}=0.96$ Positiva muy fuerte \\
\hline Sueldo & $\mathrm{r}=0.96$ Positiva muy fuerte \\
\hline Relación con los compañeros & $\mathrm{r}=0.96$ Positiva muy fuerte \\
\hline Vida personal & $\mathrm{r}=0.95$ Positiva muy fuerte \\
\hline Política y administración & $\mathrm{r}=0.85$ Positiva considerable \\
\hline Estatus & \\
\hline
\end{tabular}

Tratando de realizar una interpretación de estos resultados, podemos afirmar que casi todos los factores de desarrollo o motivadores intrínsecos al trabajo así como los factores de higiene o extrínsecos al trabajo, están presentes en la percepción (y valoración) de los trabajadores peruanos. El trabajo de investigación realizado permite deducir niveles o situaciones de satisfacción o de insatisfacción; asimismo, hubiera sido útil una medición diferenciada entre los trabajadores de Lima y los de Huancayo para indagar sobre las posibles diferencias sociales $o$ culturales dada la situación de heterogeneidad en los niveles de desarrollo entre Lima y las economías regionales.

Una primera perspectiva de análisis está referida a las prioridades ( la percepción subjetiva de lo importante).

Sobre los factores motivadores encontramos en primer lugar al Factor Responsabilidad y en ultimo lugar al Factor Logro. ¿Quiere decir esto que los trabajadores peruanos no aspiran a la autorrealización, al desarrollo personal en el sentido de Maslow-Herzberg?

Una primera respuesta sería que el grado de satisfacción de las necesidades básicas es insuficiente, lo que no permite «liberar» energías 
para ser canalizadas hacia las necesidades superiores. Esto es razonablemente comprensible en la situación concreta del mundo empresarial peruano. Una segunda respuesta estaría en las características del trabajo, del puesto laboral y del sistema de trabajo y gestión empresarial, donde no se dan las condiciones para el enriquecimiento del trabajo en la orientación de Herzberg. Para comprobar esta idea se necesitarían investigaciones cualitativas para identificar situaciones donde se pueda apreciar y comprender a cabalidad las características personales y sociales con motivación de logro,

«que se caracteriza por su seguridad personal, su disposición a aceptar más responsabilidades, el deseo de ver los resultados de su trabajo. También se inclina por aceptar ciertos riesgos si de estos depende el triunfo» ${ }^{39}$. Los trabajadores peruanos se identifican con el Factor Responsabilidad, caracterizado como la "persona que asume con compromiso y entereza la ejecución de la tarea y se siente motivada cuando le delegan responsabilidades. Le motiva ser 'dueño' de su trabajo» ${ }^{40}$.

En los Factores de Higiene los trabajadores otorgan gran importancia a los factores de seguridad y de relación con el jefe. Sobre el Factor Seguridad

«a la persona le motiva estar protegida contra el peligro o privación, cubierta de contingencias futuras. Requiere sentir seguridad en el futuro, estar libre de peligros y vivir en un ambiente agradable para él y para su familia» ${ }^{41}$.

El factor Relación con el Jefe

"se considera que el éxito laboral estará condicionado por el grado de aceptación o rechazo al jefe. A los trabajadores les interesa la responsabilidad con el jefe» ${ }^{42}$.

Sobre lo primero la inestabilidad y precariedad del empleo se manifiesta en la búsqueda de protección $\mathrm{y}$, lo segundo, manifiesta la predominancia de relaciones sociales verticales, expresión de las características organizacionales predominantes en nuestra realidad empresarial.

Un elemento a considerar en esta interpretación es el juicio del mismo Herzberg sobre los deseos y aspiraciones de los empleados relacionados a las necesidades básicas las cuales, al ser satisfechas «lo único que podemos esperar es impedir la insatisfacción y un mal rendimiento, (pero las necesidades no son aisladas, los factores higiénicos) funcionan como base esencial (de los otros) y se asocia a un trato justo en la retribución, supervisión, condiciones laborales y prácticas administrativas» ${ }^{43}$.

Lo que no sucede en nuestro país, caracterizado por fuertes desigualdades en la distribución del ingreso y falta de equidad en las relaciones sociales y oportunidades económicas. Además, las vivencias del entorno (nuestro país, su historia, cultura, sociedad y economía), son parcialmente singulares y tienden a modelar (el aprendizaje de la motivación ) diferencias entre los individuos en cuanto a las necesidades que sienten, y también estructuras singulares resultantes de necesidades individuales, en razón de la multiculturalidad, heterogeneidades y asimetrías estructurales de nuestra economía y sociedad.

Las preferencias encontradas en la conciencia de los empleados peruanos nos proporcionan pistas para futuras investigaciones sobre los procesos de motivación organizados alrededor de formas de trabajo más autónomas, de tareas organizadas en grupo y donde tengan cabida y adecuada valoración la responsabilidad y el sentido del logro y una mayor articulación con las condiciones del ambiente de trabajo para propiciar desarrollo, aprendizaje y promoción.

\section{A MANERA DE CONCLUSIONES: LA MOTIVA- CIÓN Y GESTIÓN ORGANIZACIONAL EN LA ACTUALIDAD}

Todos somos plenamente conscientes que la calidad y nivel de la capacidad organizativa y de sus recursos productivos, en particular el capital humano, son factores determinantes de la competitividad a largo plazo, especialmente cuando las aplicaciones tecnológicas y el entorno competitivo cambian tan rápidamente. Por lo tanto, el reto sigue siendo mejorar el rendimiento total de las empresas.

Asimismo, en razón de que la motivación no es un concepto simple, y que los distintos enfoques y teorías ayudan a entender la complejidad y singularidad de las personas en su comportamiento o desempeño en las organizaciones empresariales, se debe prestar atención al repertorio de comportamientos de una per- 
sona y del entorno que acompaña al repertorio para mejorar el desempeño humano.

Según los planteamientos de Thomas Gilbert, padre fundador de la gestión del rendimiento de una empresa y autor de «Human Competence», el repertorio de comportamiento de una persona está compuesto por motivos (gustos, preferencias, necesidades, valores); capacidades (aptitudes físicas y mentales) y conocimiento (educación y habilidades). El respaldo del entorno, proporcionado por la organización, está compuesto por información sobre estrategias, objetivos y rendimiento actual; instrumentos (herramientas, técnicas, tecnología, método de trabajo); e incentivos (monetarios y no monetarios) ${ }^{44}$.

Una conclusión sugerida en este artículo es que la visión de la teoría bifactorial de Herzberg sobre la satisfacción intrínseca es un elemento importante, pero sola no tiene la fuerza suficiente para explicar los procesos de la conducta laboral, tiene que integrarse a otros factores de la motivación y del entorno laboral. Por lo tanto, su hipótesis de trabajo de que únicamente los factores intrínsecos del contenido del trabajo, pueden desencadenar de manera permanente la conducta de los individuos en las empresas requiere mayor estudios para validarse en la práctica empresarial actual.

Diversos trabajos teóricos recientes proponen una integración de las teorías de la motivación, teniendo como base su real complementariedad y con el objeto de la construcción del conocimiento de un tema de tanta importancia para la sociedad y las empresas. Se reconoce el aporte de las teorías de las necesidades sobre los factores que originan los comportamientos individuales sin embargo, son limitaciones su pretensión de universalidad de los factores encontrados en sus trabajos empíricos, su carácter simplificador de dichos factores y su explicación bastante parcial de los procesos motivacionales. Mas allá de controversias, se trata de la elaboración de modelos teóricos integradores tal como se viene haciendo en los últimos años, uniendo en forma coherente distintas teorías de la motivación ${ }^{45}$.

En la actualidad en Estados Unidos, Canadá y Europa se aplica el high involvement workplace y de high perfomance wokplace; el primero más orientado a la participación en equipos semiautónomos, en industrias de alta tec- nología como sistemas automatizados, metalurgia, papel, producción de electricidad, en ciertas industrias electrónicas, y automotrices como Satum de General Motors. El segundo concepto contiene más tipos de incentivos individuales relacionados a equipos de proyectos especiales multifuncionales para distintos fines como identificación y resolución de problemas, reducción de costes, desarrollo de nuevos productos, beneficios económicos, primas, etc. La empresa actual no necesita simplemente más grupos, sino más grupos de alto rendimiento.

En el mundo de las prácticas empresariales y de la gestión de recursos humanos, el centro de la cuestión no es la satisfacción o insatisfacción laboral ni tampoco concebir solo al individuo (y su personalidad) en la organización; se trata ahora de analizar las tareas, los niveles de autonomía, las perspectivas de desarrollo personal y humano, las recompensas psicológicas de la participación en equipos, las relaciones de poder. No es suficiente la búsqueda del enriquecimiento del trabajo; ahora, distintas orientaciones innovadoras plantean la Calidad de la Vida Laboral, aún cuando el enriquecimiento del puesto siga siendo una estrategia que tiene como objeto proporcionar al trabajador más autonomía y responsabilidad.

\section{NOTAS}

Koontz, Harold y Welhrich, Heintz. Elementos de administración. Enfoque internacional.Sexta Edición. México, Mc Graw-Hill, 2002, pp. 300.

2 Roussel, Patrice. La Motivation au travail-Concept et theories, Note $\mathrm{N}^{\circ} 326$. Universite Toulouse I-Sciences Sociales, octubre 2000, p. 5 Otras definiciones: «Es el proceso por el cual la necesidad insatisfecha de una persona genera energía y dirección hacia cierto objetivo cuyo logro se supone habrá de satisfacer la necesidad» (Salazzati. Management, funciones y estilos de desarrollo. Ediciones Macchi, Buenos Aires, 1991); «Es el flujo que de por sí es un placer. Son las actividades que nos gustan, nos atraen porque al realizarlas entramos en flujo» (Goleman, Daniel. La Inteligencia Emocional en la Empresa, Ediciones. Buenos Aires, Vergara, 1999).

3 Koontz, Harold y Welhrich, Heintz. Op. cit, p. 301.

4 Arana, Walter. Diseño y validación de un modelo para la identificación y medición de los factores motivacionales de los trabajadores según la Teoría de Frederick Herzberg. Tesis para obtener el grado de Magíster en Administración, Unidad de Postgrado, Facultad de Ciencias Administrativas. Febrero 2004.

5 Las obras más importantes de F. Herzberg son: Herzberg F.; Mausner, B. y Capwell, R.O. Job attitudes: Review of research and opinion. Psichological Services of Pittsburg, 1957. Herzberg, F., Mausner, B. y Snydermann, B.B., The motivation to work. New York, John Wiley, 1959. Herzberg F. Le travail et la nature de l'homme. París, EME, 1971. 
Koontz, Harold y Welhrich, Heintz. Elementos de Administration. Enfoque Internacional. Sexta Edición. México, Mc Graw-Hill, 2002, p. 307.

6 Arana, Walter, Op. cit.

7 Stoner, James y otros. Op. cit., pp. 507-508; 490.

8 Stoner, James y otros. Op.cit., p. 496

9 Maslow, Abraham. «Una teoría de la Motivación Humana». En Hitos en la Gestión Empresarial. España, McKinsey \& Company Ediciones Apóstrofe, 1994, pp. 225-245.

10 Maslow, Abraham. Op. cit., p. 244.

11 Idem.

12 Maslow, Abraham. Op. cit., p. 241.

13 Maslow, Abraham. Op. cit., p. 243.

14 Maslow, Abraham. Op. cit., pp. 236 y 243.

15 Lawder, Edward y Suttle, Lloyd. "A causal correlation test of the Need-Hierarchy Concept». Organization Behavior and Human Performance, abril de 1972, pp. 265-287, citado en Koontz, Harold y Welhrich, Heintz. Op. cit, pp. 306.

16 Hill, Charles. Negocios Internacionales. México, Irwin/ Mcgraw-Hill, 2001. Capítulo III: Diferencias culturales, pp. 106-111 y Daniels, John y Radebaugh, Lee. Negocios Internacionales. Octava Edición. México, Pearson Educación, 2000, Capítulo II, pp. 72-76.

17 Koontz, Harold y Welhrich, Heintz. Op. cit., pp. 306.

18 Herzberg, Frederick. "Una vez más: Cómo se motiva a los empleados», en Hitos de la Gestión Empresarial. Op. cit., pp. 147-185.

19 Herzberg, Frederick. Op. cit., pp. 153-153.

20 Roussel, Patrice. Op. cit., p. 6.

21 Herzberg, Frederick. Op. cit., p. 153.

22 Drucker, Peter. La Disciplina de la innovación en Fundación Drucker; de Líder a Líder. España, Gránica, 2002, pp. 73-89. En cuanto al aprendizaje y calidad de motivos podemos distinguir entre el aprendizaje operativo (adquisición de un conocimiento o habilidad técnica y el aprendizaje estructural -capacidad de autocontrol descubierta por Aristóteles quien le dio el nombre de virtud-que orienta a la personal a su realización, al mejor logro futuro de resultados trascendentes. Una reflexión en este sentido se encuentra en Ferreiro, Pablo y Alcazar, Manuel. Gobierno de Personas en la Empresa. Lima, Escuela de Dirección, Universidad de Piura, 2001.

23 Herzberg, Frederick: Op. cit., p. 153.

24 Schwab, Donald. La motivación en el seno de una organización. En Océano-Centrum / Enciclopedia del Management, Océano Grupo Editorial, 1985, p. 763.

25 Del Castillo, Mancebo. El Administrador y su entorno dentro de la organización. México, Limusa / Grupo Noriega Editores, 1992, pp. 340-341

26 Covey, Stephen. Los hábitos de una Organización Eficiente. en Fundación Drucker. Op. cit., pp. 252-253.
27 Herzberg, Frederick, 1969. Op. cit., pp. 153-154. Una presentación de estudios críticos o confirmatorios de la teoría de Herzberg la encontramos en Dessler, Gary. Organización y Administración, Prentice Hall, 1987.

28 Herzberg, Frederick. Op. cit., p. 156.

29 W. Chan Kim y Mauborgne, Renee. Proceso justo: la administración en la economía del conocimiento. Julio-agosto de 1997. En Magretta Joan. La Administración en la Nueva Economía, Nuevas perspectivas. México, Oxford University Press, 2001, pp. 137-158.

30 Sistema sociotécnico de Tavistock. Diversos estudios elaborados por miembros del British Tavistock Institute pusieron en evidencia que existe interacción e interdependencia entre los sistemas tecnológico y social. Asimismo, se comprobó que la integración del grupo de trabajo es un factor determinante de la productividad y que no bastan las mejoras técnicas para obtener mayores rendimientos, ya que incluso cualquier mejora técnica introducida puede resultar contraproducente si afecta negativamente las relaciones sociales y grupales prevalecientes. A partir de estas conclusiones, los investigadores elaboraron el concepto de sistema sociotécnico.

31 Austin, James. El lado invisible del liderazgo. En Fundación Drucker, Op. cit., p. 498.

32 Herzberg, Frederick. Op. cit., pp. 164-165.

33 Whitsett, David. Ampliación y enriquecimiento del puesto de trabajo. En Océano, Enciclopedia del Managemet, pp. 12111214.

34 Koontz, Harold y Welhrich, Heintz . Op. cit., pp. 316-317.

35 Arana, Walter. Op. cit., p. 22.

36 Arana, Walter. Op.cit., p. 23. Para un análisis de la cultura y valores en el Perú véase: Ortiz de Zevallos, Felipe. Liderazgo a la peruana, y Hernández, Max. Es otro el rostro del Perú. Lima, Trabajos de Grupo Agenda Perú.

37 Arana, Walter. Op. cit., Capítulo III, Metodología, pp. 6471.

38 Arana, Walter. Op. cit., pp. 109-111.

39 Arana, Walter. Op. cit., pp. 74-75.

40 Arana, Walter. Op. cit., p. 75.

41 Arana, Walter. Op. cit., pp. 76-77.

42 Arana, Walter. Op. cit., p. 76.

43 Herzberg, Frederick; Mausner, Barnard y Bloch Snyderman, Bárbara. The Motivation to Work. New York, John Wiley \& Sons, Inc, 1959, pp. 114-115 citado en McGregor, Douglas. El Lado Humano de la Empresa. Capítulo 4.

44 Boyett, Joseph y Boyett, Jimmie. Hablan los Gurús. Las mejores ideas de los máximos pensadores de la administración. Bogotá, Grupo Editorial Norma, 1999, p. 340.

45 Roussel, Patrice. Op. cit., pp. 15-17. 